\title{
OPTIMAL CONTROL OF CAPITAL INJECTIONS BY REINSURANCE WITH A CONSTANT RATE OF INTEREST
}

\author{
JULIA EISENBERG* AND \\ HANSPETER SCHMIDLI, ${ }^{* *}$ University of Cologne
}

\begin{abstract}
We consider a classical risk model and its diffusion approximation, where the individual claims are reinsured by a reinsurance treaty with deductible $b \in[0, \tilde{b}]$. Here $b=\tilde{b}$ means 'no reinsurance' and $b=0$ means 'full reinsurance'. In addition, the insurer is allowed to invest in a riskless asset with some constant interest rate $m>0$. The cedent can choose an adapted reinsurance strategy $\left\{b_{t}\right\}_{t \geq 0}$, i.e. the parameter can be changed continuously. If the surplus process becomes negative, the cedent has to inject additional capital. Our aim is to minimise the expected discounted capital injections over all admissible reinsurance strategies. We find an explicit expression for the value function and the optimal strategy using the Hamilton-Jacobi-Bellman approach in the case of a diffusion approximation. In the case of the classical risk model, we show the existence of a 'weak' solution and calculate the value function numerically.
\end{abstract}

Keywords: Optimal control; stochastic control; Hamilton-Jacobi-Bellman equation; capital injection; classical risk model; constant interest rate; riskless asset

2010 Mathematics Subject Classification: Primary 60K10

Secondary 91B30; 60J65

\section{Introduction}

The classical measure for an insurance risk is the ruin probability. This is the probability that the surplus process of an insurance company becomes negative in finite time. Ruin probabilities are, from the perspective of a risk manager, the natural dynamic counterpart of the value at risk. We say that ruin occurs when the surplus process, modelled as a stochastic process, becomes negative for the first time. An introduction to ruin probabilities can, for instance, be found in the books by Asmussen [1], Grandell [5], and Rolski et al. [9]. For taking decisions, a natural criterion is therefore to minimise the ruin probability. For example, an actuary may look for the reinsurance strategy that minimises the ruin probability. Numerous papers have been written on minimising the ruin probability in the Cramér-Lundberg model or its diffusion approximation. See [6], [7], [10], [11], and [13], among others.

The ruin probability indicates the soundness of the insurer's combination of the income of an insurance company plus the initial capital on the one hand and the claims process on the other. Also, we obtain a useful tool for portfolio comparison. But despite these positive points, the use of ruin probabilities has been criticised. For instance, the ruin probability does not take into account the time of ruin nor the severity of ruin.

Received 22 July 2010; revision received 16 February 2011.

* Current address: Financial and Actuarial Mathematics, Vienna University of Technology, A-1040 Vienna, Austria.

Partly supported by the Austrian science fund, grant number FWF22449.

** Postal address: Institute of Mathematics, University of Cologne, Weyertal 86-90, D-50931 Cologne, Germany.

Email address: schmidli@math.uni-koeln.de 
Eisenberg and Schmidli [3], [4] introduced an alternative measure of risk. They proposed to valuate the capital injections. Let $X$ be the underlying surplus process with $X_{0}=x$. Let $Y$ be an increasing process with $Y_{0-}=0$. The process with capital injections is denoted by $X_{t}^{Y}=X_{t}+Y_{t}$. We define the value $V^{Y}(x)=\mathrm{E}_{x}\left[\int_{0}^{\infty} \mathrm{e}^{-\delta t} \mathrm{~d} Y_{t}\right]$, where $\delta \geq 0$. The injection process $Y$ has to be chosen such that $X_{t}^{Y} \geq 0$ for all $t$ (almost surely). The value function is defined as $V(x)=\inf V^{Y}(x)$, where the infimum is taken over all càdlàg processes $Y$ such that $X_{t}^{Y} \geq 0$ for all $t$. Because of the discounting or because ruin is not certain, it is not optimal to inject capital before it is really necessary.

The problem of minimising the expected discounted capital injections in the CramérLundberg model and in its diffusion approximation with dynamic reinsurance has already been solved in [3] and [4]. Optimal reinsurance strategies with respect to ruin probabilities have been considered in [10] (see also [12]), and in [2] with respect to dividends. Here we consider an extension to the above model. We allow the insurer to invest the positive excess in a riskless asset with a constant interest rate $m$. We are also interested in finding the optimal reinsurance strategy and the value function as the infimum of all possible expected discounted capital injections due to admissible reinsurance strategies.

Let $(\Omega, \mathcal{F}, \mathrm{P})$ be a complete probability space that is large enough to carry all the stochastic objects defined below. By $\mathbb{F}=\left\{\mathscr{F}_{t}: t \geq 0\right\}$ we denote the natural filtration of a Brownian motion $W$ or, accordingly, of the loss process in the Cramér-Lundberg model. The paper is organised as follows. In Section 2 we consider a diffusion approximation to the Cramér-Lundberg model, and calculate the value function and the optimal strategy explicitly. In Section 3 we consider the Cramér-Lundberg model for the case where the preference rate $\delta$ is nonnegative. Here, a closed expression for the value function is not available. The value function and the optimal strategy are calculated numerically in Subsection 3.1 for exponentially and Pareto-distributed claim sizes.

\section{Proportional reinsurance for a diffusion approximation}

Consider the surplus process of an insurance company, where the time horizon is infinite:

$$
C_{t}=x+c t-\sum_{i=1}^{N_{t}} Z_{i}
$$

Here $\left\{N_{t}\right\}$ is the Poisson process with intensity $\lambda>0$ and $\left\{Z_{i}\right\}_{i \in \mathbb{N}}$ is a sequence of independent and identically distributed random variables. The $Z_{i}$ are assumed to have a distribution $G$ with $\mu=\mathrm{E}\left[Z_{i}\right]$ and $\mu_{2}=\mathrm{E}\left[Z_{i}^{2}\right]<\infty$, and to be independent of $\left\{N_{t}\right\}$. The premium income of the insurer is $c=(1+\eta) \lambda \mu$ for some $\eta>0$. Furthermore, the insurer can buy proportional reinsurance. That is, the insurer has to choose a retention level $b \in[0,1]$ and the reinsurer carries $(1-b) Z_{i}$ from each claim $Z_{i}$. The premium rate remaining to the insurer calculated by an expected value principle is $c(b)=\lambda \mu b(1+\theta)-\lambda \mu(\theta-\eta)$, where $\theta$ is the safety loading of the reinsurer. In order to avoid the case where the insurer can get rid of the risk by buying full reinsurance and still receiving a nonnegative premium, we assume that $\theta>\eta$. The insurer can change his retention level continuously.

A diffusion approximation to the above classical risk model then fulfils the stochastic differential equation

$$
\mathrm{d} X_{t}^{B}=\left\{\lambda \mu\left[b_{t} \theta-(\theta-\eta)\right]\right\} \mathrm{d} t+b_{t} \sqrt{\lambda \mu_{2}} \mathrm{~d} W_{t},
$$


where $\left\{W_{t}\right\}$ is a standard Brownian motion. In this section we work on a probability space $(\Omega, \mathcal{F}, \mathrm{P})$ containing the Brownian motion $\left\{W_{t}\right\}$. We call the reinsurance strategy $B=\left\{b_{t}\right\}$ admissible if it is adapted and càdlàg, and $b_{t} \in[0,1]$ for all $t$; the set of all reinsurance strategies is denoted by $\mathcal{U}$. Since $b_{t}$ is bounded and càdlàg, the integrals are well defined.

Now we allow the insurer to earn interest on positive surplus with a constant force of interest. It is clear that if $X$ is at 0 , we must inject capital to stop the process entering $(-\infty, 0)$. We interpret $Y_{t}$ as the cumulative capital injections up to time $t$ and associate with $Y^{B}=\left\{Y_{t}^{B}\right\}$ the controlled process with capital injections

$$
\mathrm{d} X_{t}^{B, Y, m}=\left\{m X_{t}^{B, Y, m}+\lambda \mu\left[b_{t} \theta-(\theta-\eta)\right]\right\} \mathrm{d} t+b_{t} \sqrt{\lambda \mu_{2}} \mathrm{~d} W_{t}+\mathrm{d} Y_{t}^{B}
$$

for a constant interest rate $m>0$. Because the preference rate $\delta$ is nonnegative, we should inject capital only when the process becomes negative and only enough to allow the process to shift to 0 again. The process $Y$ is thus defined as the solution to the Skorokhod problem; see, for example, [8, p. 117]. That is, the smallest nondecreasing process $\left\{Y_{t}\right\}$ such that $X_{t}^{B, Y, m} \geq 0$ for all $t$. It is well known that the process $Y$ exists. Note that as a nondecreasing process, $Y^{B}=\left\{Y_{t}^{B}\right\}$ is of bounded variation.

We want to measure the risk, connected to some reinsurance strategy $B$, by the expected discounted capital injections $V^{B}(x):=\mathrm{E}_{x}\left[\int_{0}^{\infty} \mathrm{e}^{-\delta t} \mathrm{~d} Y_{t}^{B}\right]$. Our goal is to find the value function by minimising $V^{B}(x)$ over all admissible reinsurance strategies:

$$
V(x):=\inf _{B \in \mathcal{U}} V^{B}(x) .
$$

It would seem natural that $\delta \geq m$. Indeed, if $\delta<m$, the capital injections would be discounted at a lower rate than the surplus. However, we do not make a restriction, and allow all $\delta \geq 0$ and $m>0$.

It is clear that the value function $V(x)$ is decreasing. In particular, we obtain, for the constant strategy $B \equiv 0$ before ruin occurs,

$$
\begin{aligned}
X_{t}^{0, m} & =x-\lambda \mu(\theta-\eta) t+m \int_{0}^{t} X_{s}^{0, m} \mathrm{~d} s \\
& =\left(x-\lambda \mu(\theta-\eta) m^{-1}\right) \mathrm{e}^{m t}+\lambda \mu(\theta-\eta) m^{-1} .
\end{aligned}
$$

Since it holds that $X_{t}^{0, m}>0$ for all $t$ if $x \geq \lambda \mu(\theta-\eta)$, we conclude that $\left\{Y_{t}^{0}\right\} \equiv 0$. Therefore, $V(x)=0$ for $x \geq \lambda \mu(\theta-\eta)$. Thus, we have to consider only $0 \leq x<\lambda \mu(\theta-\eta) m^{-1}$.

Remark 2.1. Let $\left\{X_{t}\right\}$ be a process fulfilling the stochastic differential equation

$$
\mathrm{d} X_{t}=a\left(X_{t}\right) \mathrm{d} t+\sigma\left(X_{t}\right) \mathrm{d} W_{t},
$$

where $a$ and $\sigma$ are functions such that the above equation has a unique strong solution. The process with capital injections then fulfils

$$
\mathrm{d} X_{t}^{Y}=a\left(X_{t}^{Y}\right) \mathrm{d} t+\sigma\left(X_{t}^{Y}\right) \mathrm{d} W_{t}+\mathrm{d} Y_{t},
$$

whereas $Y$ is the local time of the process at 0 .

Shreve et al. [14] showed that the corresponding return function $V(x)=\mathrm{E}_{x}\left[\int_{0}^{\infty} \mathrm{e}^{-\delta t} \mathrm{~d} Y_{t}\right]$ solves the differential equation

$$
\frac{\sigma^{2}(x)}{2} V^{\prime \prime}(x)+a(x) V^{\prime}(x)-\delta V(x)=0 \quad \text { for } x \geq 0,
$$


and fulfils $V^{\prime}(0)=-1$ and $\lim _{x \rightarrow \infty} V(x)=0$. From Shreve et al. [14] we also know that every solution $f(x)$ to the above differential equation, vanishing at $\infty$, has the form

$$
f(x)=f^{\prime}(0) \mathrm{E}_{x}\left[\int_{0}^{\infty} \mathrm{e}^{-\delta t} \mathrm{~d} Y_{t}\right] .
$$

Now equipped with the knowledge of how to calculate the return function for a given reinsurance strategy $B$, we illustrate the method by an example.

Example 2.1. Consider a constant strategy $B \equiv b \in[0,1]$. Owing to Remark 2.1, the corresponding return function $V^{b}(x)$ solves the differential equation

$$
\frac{b^{2} \lambda \mu_{2}}{2} f^{\prime \prime}(x)+(m x+\lambda \mu(b \theta-(\theta-\eta))) f^{\prime}(x)-\delta f(x)=0 .
$$

With the power series method we find that solutions to the above differential equation are given by

$$
\begin{aligned}
C_{1}\left(1+\sum_{n=1}^{\infty}\right. & \left.\frac{\prod_{k=1}^{n}(\delta / m+2-2 k)}{(2 n) !}\left(\frac{2 m}{\lambda \mu_{2} b^{2}}\right)^{n}\left(x+\lambda \mu(b \theta-\theta+\eta) m^{-1}\right)^{2 n}\right) \\
+ & C_{2}\left(\sum_{n=1}^{\infty} \frac{\prod_{k=1}^{n}(\delta / m+1-2 k)}{(2 n+1) !}\left(\frac{2 m}{\lambda \mu_{2} b^{2}}\right)^{n}\left(x+\lambda \mu(b \theta-\theta+\eta) m^{-1}\right)^{2 n+1}\right. \\
& \left.+x+\lambda \mu(b \theta-\theta+\eta) m^{-1}\right) .
\end{aligned}
$$

Using the initial conditions $\lim _{x \rightarrow \infty} V^{b}(x)=0$ and $\left(V^{b}\right)^{\prime}(0)=-1$, we can calculate the coefficients $C_{1}$ and $C_{2}$.

Let, for example, $b=0.5, \lambda=\mu=1, \mu_{2}=2, \theta=0.5, \eta=0.3, \delta=0.04$, and $m=0.03$. Then we obtain

$$
C_{1}=4.084921164 \text { and } C_{2}=-1.947322694 .
$$

Changing the parameter $\theta$ to $\theta=0.8$ yields

$$
C_{1}=0.9686572638 \text { and } C_{2}=-0.4617685869 .
$$

In Figure 1 we plot the return functions for the constant strategy $B \equiv 0.5, V_{0.5}^{0.5}(x)$ for $\theta=0.5$ (solid line) and $V_{0.8}^{0.5}(x)$ for $\theta=0.8$ (dotted line), and the return function for $B \equiv 1, V^{1}(x)$ (dashed line). We see that, for $\theta=0.5$, the return function corresponding to $B \equiv 0.5$ lies below $V^{1}(x)$, and, for $\theta=0.8$, above $V^{1}(x)$. We will see later that, for some $\theta$, it holds that $V^{1}(x)=V(x)$ on some intervals.

Recall that $V(x)=0$ for $x \geq \lambda \mu(\theta-\eta) m^{-1}$. For $x<\lambda \mu(\theta-\eta) m^{-1}$, the HamiltonJacobi-Bellman (HJB) equation is given by

$$
\inf _{b \in[0,1]} \frac{1}{2} \lambda \mu_{2} b^{2} V^{\prime \prime}(x)+\{m x-\lambda \mu(\theta-\eta)+\lambda \mu b \theta\} V^{\prime}(x)-\delta V(x)=0 .
$$

We abandon the explicit derivation of the HJB equation. Note that if the value function is twice continuously differentiable and solves the HJB equation above, it must be strictly convex. In fact, choosing $\hat{b}=1-\eta / \theta-m x / \lambda \mu \theta$ (note that $\hat{b} \in[0,1]$ ) we obtain

$$
\frac{1}{2} \lambda \mu_{2} \hat{b}^{2} V^{\prime \prime}(x)-\delta V(x) \geq 0 .
$$

Since $V(x)>0$, convexity follows. 


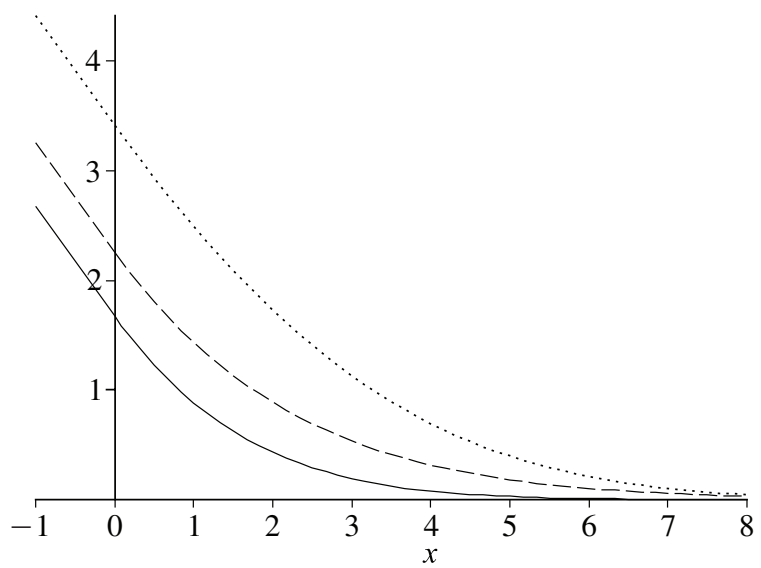

FIGURE 1: Return functions $V_{0.5}^{0.5}(x)$ (solid line), $V_{0.8}^{0.5}(x)$ (dotted line), and $V^{1}(x)$ (dashed line).

We make the ansatz

$$
V(x)=C\left(m^{-1} \lambda \mu(\theta-\eta)-x\right)^{\kappa}
$$

for some $C>0$ and $\kappa>1$. Then (2.2) reads

$$
\begin{aligned}
0=\inf _{b \in[0,1]}\left\{\frac{1}{2} \lambda \mu_{2} b^{2} \kappa(\kappa-1)\left(m^{-1} \lambda \mu(\theta-\eta)-x\right)^{\kappa-2}\right. \\
-\{m x+\lambda \mu[b \theta-(\theta-\eta)]\} \kappa\left(m^{-1} \lambda \mu(\theta-\eta)-x\right)^{\kappa-1} \\
\left.-\delta\left(m^{-1} \lambda \mu(\theta-\eta)-x\right)^{\kappa}\right\} .
\end{aligned}
$$

The optimal $b$ is then given by

$$
b(x)=\frac{\theta \mu\left(m^{-1} \lambda \mu(\theta-\eta)-x\right)}{\mu_{2}(\kappa-1)},
$$

provided that $b(x) \leq 1$, i.e. $x$ is close enough to $m^{-1} \lambda \mu(\theta-\eta)$. If $b(x)>1$, no reinsurance has to be chosen.

Plugging in the optimal $b(x)$ and dividing by $\left(m^{-1} \lambda \mu(\theta-\eta)-x\right)^{\kappa}$, we find that

$$
m \kappa-\frac{\lambda \kappa \theta^{2} \mu^{2}}{2 \mu_{2}(\kappa-1)}-\delta=0 .
$$

Solving for $\kappa$ yields the solution

$$
\kappa=\frac{\delta \mu_{2}+m \mu_{2}+\lambda \theta^{2} \mu^{2} / 2+\sqrt{\left(\delta \mu_{2}+m \mu_{2}+\lambda \theta^{2} \mu^{2} / 2\right)^{2}-4 m \mu_{2}^{2} \delta}}{2 m \mu_{2}} .
$$

Note that the other solution is smaller than 1.

Remark 2.2. Let $X^{*}=X^{b(x), Y, m}$ with initial value $0 \leq x<\lambda \mu(\theta-\eta) m^{-1}$, where the reinsurance strategy $b(x)$ is given in (2.3). Consider the process $Z_{t}=m^{-1} \lambda \mu(\theta-\eta)-X_{t}^{*}$ for $t \in\left[0, \tau^{*}\right]$, where $\tau^{*}=\inf \left\{s: X_{s}^{*}=0\right\}$. Then

$$
\mathrm{d} Z_{t}=-\frac{\sqrt{\lambda \mu_{2}} \theta \mu}{\mu_{2}(\kappa-1)} Z_{t} \mathrm{~d} W_{t}-\left(\frac{\lambda \mu^{2} \theta^{2}}{\mu_{2}(\kappa-1)}-m\right) Z_{t} \mathrm{~d} t
$$


This means that $\left\{Z_{t}\right\}$ is a geometric Brownian motion. Taking the logarithm gives

$$
\mathrm{d}\left(\log \left(Z_{t}\right)\right)=-\frac{\sqrt{\lambda \mu_{2}} \theta \mu}{\mu_{2}(\kappa-1)} \mathrm{d} W_{t}+\left(m-\frac{\lambda \mu^{2} \theta^{2}(2 \kappa-1)}{2 \mu_{2}(\kappa-1)^{2}}\right) \mathrm{d} t .
$$

In particular, the surplus $X^{*}$ will never reach the value $m^{-1} \lambda \mu(\theta-\eta)$, where full reinsurance would be bought.

The considerations we used in deriving (2.3) are of a heuristic nature. Hence, it remains to prove the verification theorem.

Theorem 2.1. (Verification theorem.) Define $\tilde{x}:=\left\{m^{-1} \lambda \mu(\theta-\eta)-\mu_{2}(\kappa-1) / \theta \mu\right\} \vee 0$. Then the strategy

$$
b^{*}(x)= \begin{cases}0, & x \geq m^{-1} \lambda \mu(\theta-\eta), \\ b(x), & \tilde{x}<x<m^{-1} \lambda \mu(\theta-\eta), \\ 1, & x \leq \tilde{x},\end{cases}
$$

where $b(x)$ is given in (2.3), is an optimal reinsurance strategy. The function $f(x)$, given by

$$
f(x)= \begin{cases}0, & x \geq m^{-1} \lambda \mu(\theta-\eta), \\ f_{2}(x), & \tilde{x} \leq x<m^{-1} \lambda \mu(\theta-\eta), \\ f_{1}(x), & 0<x<\tilde{x} \\ f(0)-x, & x \leq 0\end{cases}
$$

with

$$
\begin{aligned}
f_{1}(x)= & C_{1}\left(1+\sum_{n=1}^{\infty} \frac{\prod_{k=1}^{n}(\delta / m+2-2 k)}{(2 n) !}\left(\frac{2 m}{\lambda \mu_{2}}\right)^{n}\left(x+\lambda \mu \eta m^{-1}\right)^{2 n}\right) \\
& +C_{2}\left(x+\lambda \mu \eta m^{-1}+\sum_{n=1}^{\infty} \frac{\prod_{k=1}^{n}(\delta / m+1-2 k)}{(2 n+1) !}\left(\frac{2 m}{\lambda \mu_{2}}\right)^{n}\left(x+\lambda \mu \eta m^{-1}\right)^{2 n+1}\right)
\end{aligned}
$$

and

$$
f_{2}(x)=C_{3}\left(r^{-1} \lambda \mu(\theta-\eta)-x\right)^{\kappa},
$$

where $\kappa$ is given in (2.5), is twice continuously differentiable, solves the HJB equation (2.2), and $f(x)=V(x)$. If $\tilde{x}>0$, the coefficients $C_{1}, C_{2}$, and $C_{3}$ are uniquely determined by the system of equations

$$
f_{1}^{\prime}(0)=-1, \quad f_{1}^{\prime}(\tilde{x})=f_{2}^{\prime}(\tilde{x}), \quad f_{1}^{\prime \prime}(\tilde{x})=f_{2}^{\prime \prime}(\tilde{x})
$$

if $\tilde{x} \leq 0, C_{3}$ is given by $f_{2}^{\prime}(0)=-1$.

Proof. We have already seen that $f(x)$ solves the HJB equation (2.2) in the interval $(\hat{x}$, $\left.m^{-1} \lambda \mu(\theta-\eta)\right)$. From Example 2.1 we know that $f_{1}(x)$ solves $(2.2)$ provided that $b^{*}(x)=1$. It therefore remains to show that the infimum really is attained at $b^{*}(x)=1$ for $x \in[0, \tilde{x}]$. Note that since $f_{1}^{\prime}(\tilde{x})=f_{2}^{\prime}(\tilde{x})$ and $f_{1}^{\prime \prime}(\tilde{x})=f_{2}^{\prime \prime}(\tilde{x})$, we have $b^{*}(\tilde{x})=1$.

Assume that $\tilde{x}>0$, otherwise there is nothing to show. Note that $\lambda \mu m^{-1}(\theta-\eta)-$ $\mu_{2}(\kappa-1) / \theta \mu \geq 0$ holds if and only if

$$
\eta \leq \theta \frac{1-2 \delta / m \kappa}{2(1-\delta / m \kappa)}
$$


Consider the HJB equation (2.2) with the function $f_{1}(x)$. Since the minimum is attained at

$$
b^{*}(x)=\frac{-\mu \theta f_{1}^{\prime}(x)}{\mu_{2} f_{1}^{\prime \prime}(x)} \wedge 1,
$$

we need to show that $g(x):=-\mu \theta f_{1}^{\prime}(x) / \mu_{2} f_{1}^{\prime \prime}(x) \geq 1$ for all $x \in[0, \tilde{x}]$.

Assume for the moment that there exists some $x \in[0, \tilde{x}]$ with $g(x)<1$. Because $g(\tilde{x})=1$ and $g$ is continuous, there exist some interval $[a, b] \subset[0, \tilde{x}]$ and $x^{*} \in[a, b]$ such that $g^{\prime}(x)>0$ on $[a, b]$ and $g\left(x^{*}\right)<1$. So we know that

$$
g^{\prime}\left(x^{*}\right)=-\frac{\theta \mu}{\mu_{2}}+\frac{\theta \mu}{\mu_{2}} \frac{f^{\prime}\left(x^{*}\right) f^{\prime \prime \prime}\left(x^{*}\right)}{f^{\prime \prime}\left(x^{*}\right)^{2}}>0 .
$$

It readily follows that

$$
1<\frac{f_{1}^{\prime}\left(x^{*}\right) f_{1}^{\prime \prime \prime}\left(x^{*}\right)}{f_{1}^{\prime \prime}\left(x^{*}\right)^{2}}=\frac{-\theta \mu f_{1}^{\prime}\left(x^{*}\right)}{\mu_{2} f_{1}^{\prime \prime}\left(x^{*}\right)} \frac{-\mu_{2} f_{1}^{\prime \prime \prime}\left(x^{*}\right)}{\theta \mu f_{1}^{\prime \prime}\left(x^{*}\right)}=g\left(x^{*}\right) \frac{-\mu_{2} f_{1}^{\prime \prime \prime}\left(x^{*}\right)}{\theta \mu f_{1}^{\prime \prime}\left(x^{*}\right)} .
$$

Because $g\left(x^{*}\right)$ was assumed to be smaller than $1,-\mu_{2} f_{1}^{\prime \prime \prime}\left(x^{*}\right) / \theta \mu f_{1}^{\prime \prime}\left(x^{*}\right)$ has to be larger than 1.

The function $f_{1}(x)$ is smooth. From Example 2.1 we know that $f_{1}(x)$ fulfils the differential equation

$$
\frac{\lambda \mu_{2}}{2} f_{1}^{\prime \prime}(x)+(m x+\lambda \mu \eta) f_{1}^{\prime}(x)-\delta f_{1}(x)=0,
$$

from which we obtain the following representation:

$$
\frac{\lambda \mu_{2}}{2} f_{1}^{\prime \prime \prime}\left(x^{*}\right)+\left\{m x^{*}+\lambda \mu \eta\right\} f_{1}^{\prime \prime}\left(x^{*}\right)-(\delta-m) f_{1}^{\prime}\left(x^{*}\right)=0 .
$$

Rearranging the terms, dividing by $f_{1}^{\prime \prime}\left(x^{*}\right)$, and using (2.4) yields, for $\delta \geq m$,

$$
\begin{aligned}
\frac{-\mu_{2} f_{1}^{\prime \prime \prime}\left(x^{*}\right)}{\theta \mu f_{1}^{\prime \prime}\left(x^{*}\right)} & =-(\delta-m) \frac{2 f_{1}^{\prime}\left(x^{*}\right)}{\lambda \theta \mu f_{1}^{\prime \prime}\left(x^{*}\right)}+\frac{2}{\lambda \theta \mu}\left\{m x^{*}+\lambda \mu \eta\right\} \\
& <(\delta-m) \frac{2 \mu_{2}}{\lambda \theta^{2} \mu^{2}}+\frac{2}{\lambda \theta \mu}\{m \tilde{x}+\lambda \mu \eta\} \\
& =(\delta-m) \frac{2 \mu_{2}}{\lambda \theta^{2} \mu^{2}}+\frac{2}{\lambda \theta \mu}\left\{\lambda \mu \theta-\frac{m \mu_{2}(\kappa-1)}{\theta \mu}\right\} \\
& =2-\frac{\kappa}{\kappa-1} \\
& =\frac{\kappa-2}{\kappa-1} \\
& <1
\end{aligned}
$$

where we used the definition of $\kappa$. This is a contradiction. For $m>\delta$, we also obtain a contradiction. Because $f_{1}^{\prime}(x) \leq 0$ and $f_{1}^{\prime \prime}(x) \geq 0$, we have, from the definition of $\kappa$,

$$
\frac{-\mu_{2} f_{1}^{\prime \prime \prime}\left(x^{*}\right)}{\theta \mu f_{1}^{\prime \prime}\left(x^{*}\right)}<2-\frac{m \kappa}{m \kappa-\delta}=\frac{m \kappa-2 \delta}{m \kappa-\delta}<1 .
$$


Now we will show that $f(x)=V(x)$. Consider an arbitrary admissible reinsurance strategy $B=\left\{b_{t}\right\}$, and define $\hat{X}_{t}=X^{B, Y, m}$. Then $\hat{X}$ is given by the differential equation

$$
\mathrm{d} \hat{X}_{t}=\left\{m \hat{X}_{t}+\lambda \mu\left[b_{t} \theta-(\theta-\eta)\right]\right\} \mathrm{d} t+b_{t} \sqrt{\lambda \mu_{2}} \mathrm{~d} W_{t}+\mathrm{d} Y_{t}^{B} .
$$

Let $x_{n}=m^{-1} \lambda \mu(\theta-\eta)-n^{-1}$. We suppose that $n$ is large enough that $x_{n}>\tilde{x}$. Furthermore, let $\tau_{n}=\inf \left\{t: \hat{X}_{t}>x_{n}\right\}$ and $\tau_{0}=\lim _{n \rightarrow \infty} \tau_{n}=\infty$; see Remark 2.2. Since $f(x)$ is twice continuously differentiable, $\hat{X}_{t} \geq 0$, and $f^{\prime}(0)=-1$, using (2.2), we apply Itô's formula to the function $\mathrm{e}^{-\delta t} f(x)$ to obtain

$$
\begin{aligned}
\mathrm{e}^{-\delta\left(\tau_{n} \wedge t\right)} f\left(\hat{X}_{\tau_{n} \wedge t}\right)= & f(x)+\int_{0}^{\tau_{n} \wedge t} \mathrm{e}^{-\delta s} f^{\prime}\left(\hat{X}_{s}\right) \mathrm{d} Y_{s}^{B} \\
& +\int_{0}^{\tau_{n} \wedge t} \mathrm{e}^{-\delta s}\left\{D_{s, B} f\left(\hat{X}_{s}\right)-\delta f\left(\hat{X}_{s}\right)\right\} \mathrm{d} s \\
& +\int_{0}^{\tau_{n} \wedge t} \mathrm{e}^{-\delta s} f^{\prime}\left(\hat{X}_{s}\right) b_{s} \sqrt{\lambda \mu_{2}} \mathrm{~d} W_{s} \\
\geq & f(x)+\int_{0}^{\tau_{n} \wedge t} \mathrm{e}^{-\delta s} \mathrm{~d} Y_{s}^{B} \\
& +\int_{0}^{\tau_{n} \wedge t} \mathrm{e}^{-\delta s} f^{\prime}\left(\hat{X}_{s}\right) b_{s} \sqrt{\lambda \mu_{2}} \mathrm{~d} W_{s},
\end{aligned}
$$

where

$$
D_{s, B} f(x)=\frac{\lambda \mu_{2} b_{s}^{2}}{2} f^{\prime \prime}(x)+\left\{m x+\lambda \mu\left(b_{s} \theta-\theta+\eta\right)\right\} f^{\prime}(x)
$$

is the infinitesimal generator of the process $X_{t}^{b_{s}, m}$. Because the derivative of the value function is bounded, we can conclude that the stochastic integral is a martingale with zero expectation. Thus, taking expectations of both sides of the above inequality we have

$$
f(x) \leq \mathrm{E}_{x}\left[\mathrm{e}^{-\delta\left(\tau_{n} \wedge t\right)} f\left(\hat{X}_{\tau_{n} \wedge t}\right)\right]+\mathrm{E}_{x}\left[\int_{0}^{\tau_{n} \wedge t} \mathrm{e}^{-\delta s} \mathrm{~d} Y_{s}^{B}\right] .
$$

Letting $n \rightarrow \infty$ we obtain, using the fact that $f\left(\hat{X}_{\tau_{0}}\right)=0$,

$$
f(x) \leq \mathrm{e}^{-\delta t} \mathrm{E}_{x}\left[f\left(\hat{X}_{t}\right) ; \tau_{0}>t\right]+\mathrm{E}_{x}\left[\int_{0}^{\tau_{0} \wedge t} \mathrm{e}^{-\delta s} \mathrm{~d} Y_{s}^{B}\right] .
$$

Since $f(x)$ is bounded, we can let $t \rightarrow \infty$, yielding

$$
f(x) \leq \mathrm{E}_{x}\left[\int_{0}^{\tau_{0}} \mathrm{e}^{-\delta s} \mathrm{~d} Y_{s}^{B}\right] \leq \mathrm{E}_{x}\left[\int_{0}^{\infty} \mathrm{e}^{-\delta s} \mathrm{~d} Y_{s}^{B}\right] .
$$

This implies that $f(x) \leq V(x)$. Repeating the calculations above with the proposed optimal strategy, the inequalities become equalities. This proves that $f(x)=V(x)$.

Example 2.2. Consider the parameters $\eta=0.3, \lambda=\mu=1, \mu_{2}=2, \delta=0.04, \theta=0.8$, and $m=0.03$. It is easy to verify that $\kappa=7.49, \tilde{x}=0.441 \overline{6}, \lambda \mu(\theta-\eta) m^{-1}=16 . \overline{6}$, and that the 

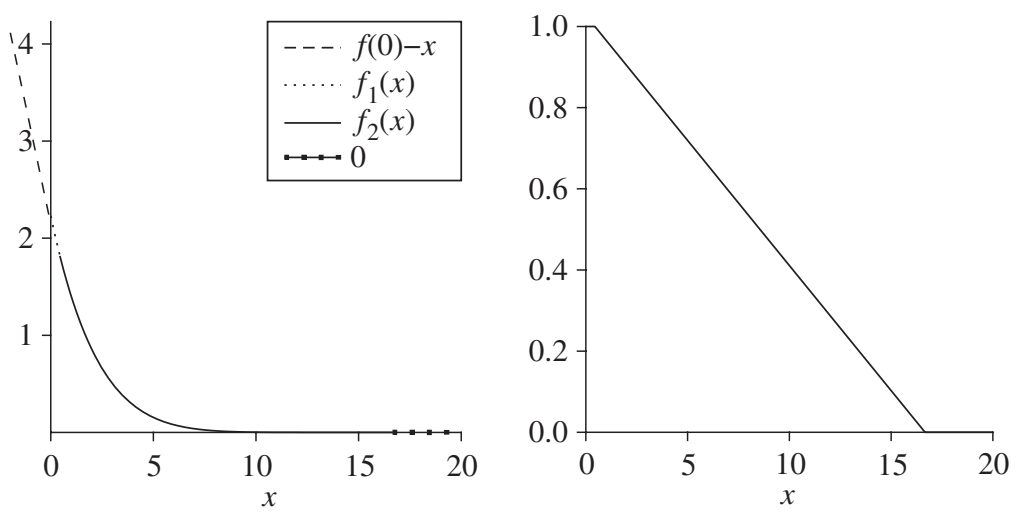

Figure 2: The value function $V(x)(l e f t)$ and the optimal strategy $b^{*}(x)(r i g h t)$.

optimal strategy on $[0.441 \overline{6}, 16 . \overline{6}]$ and the value function are given by

$$
\begin{aligned}
b(x)= & \frac{13.3-0.8 x}{12.98}, \\
f_{1}(x)= & 64.28\left(1+\sum_{n=1}^{\infty} \frac{1.33 \cdots(1.33-2 n+2)}{(2 n) !} 0.03^{n}(x+10)^{2 n}\right) \\
& -15.32\left(x+10+\sum_{n=1}^{\infty} \frac{(1.33-1) \cdots(1.33-2 n+1)}{(2 n+1) !} 0.03^{n}(x+10)^{2 n+1}\right), \\
f_{2}(x)= & 1.575819495 \cdot 10^{-9}(6.7-x)^{7.49} .
\end{aligned}
$$

The optimal strategy and the value function are given in Figure 2. In the left picture we can see the value function, composed of four functions. The dashed line corresponds to $f(0)-x$, the dotted line to $f_{1}(x)$, the solid line to $f_{2}(x)$, and the line with squares to 0 .

\section{The classical risk model}

In this section we consider the classical risk model $(2.1)$. The probability space $(\Omega, \mathcal{F}, \mathrm{P})$ is assumed to contain the compound Poisson process $\sum_{i=1}^{N_{t}} Z_{i}$. By $Z$ we denote a generic random variable with the same distribution as $Z_{i}$. The insurer can buy reinsurance. In contrast to the previous section, we now allow more general reinsurance treaties. In the examples we will again return to proportional reinsurance. Choosing the level $b \in[0, \tilde{b}]$, the insurer pays $r\left(Z_{i}, b\right)$ for a claim of size $Z_{i}$. Here $b=0$ means 'full reinsurance' and $b=\tilde{b} \in(0, \infty]$ means 'no reinsurance'. For example, for proportional reinsurance, $r(Z, b)=b Z$ and $b \in[0,1]$. For excess of loss reinsurance, we obtain $r(Z, b)=\min \{Z, b\}$ and $b \in[0, \infty]$.

For the reinsurance cover, the insurer pays a premium at rate $c-c(b)$. That is, the premium rate left for the cedent is $c(b)$. For simplicity, we assume that $r(z, b)$ is continuous and increasing in both $z$ and $b$, and that $c(b)$ is continuous and increasing with $c(0)<0$ and $c(\tilde{b})=c$. The assumption that $c(0)<0$ is needed in order that the problem below is not trivial. For example, if the reinsurer uses an expected value principle with safety loading $\theta$, we obtain

$$
c(b)=c-(1+\theta) \lambda \mathrm{E}[Z-r(Z, b)]=(1+\theta) \lambda \mathrm{E}[r(Z, b)]-(\theta-\eta) \lambda \mu .
$$


The condition $c(0)<0$ is fulfilled if $\theta>\eta$. The insurer can choose the level $b_{t}$ at any time point $t$. Because no information about the future can be used, the process $\left\{b_{t}\right\}$ is assumed to be càdlàg and adapted. The surplus of the insurer including reinsurance then has the form

$$
X_{t}^{B}=x+\int_{0}^{t} c\left(b_{s}\right) \mathrm{d} s-\sum_{i=1}^{N_{t}} r\left(Z_{i}, b_{T_{i}-}\right)
$$

As before, the insurer can invest his money, if his surplus is positive, with a fixed rate of interest $m>0$. To prevent the surplus process becoming negative, the insurer has to inject additional capital. We denote the accumulated capital injections until time $t$ by $\left\{Y_{t}^{B}\right\}$. The surplus process therefore has the form

$$
X_{t}^{B, m, Y}=x+\int_{0}^{t}\left(c\left(b_{s}\right)+m X_{s}^{B, m, Y}\right) \mathrm{d} s-\sum_{i=1}^{N_{t}} r\left(Z_{i}, b_{T_{i}-}\right)+Y_{t}^{B} .
$$

We are interested in the minimal value $V(x)=\inf _{B \in U} \mathrm{E}\left[\int_{0}^{\infty} \mathrm{e}^{-\delta t} \mathrm{~d} Y_{t}^{B}\right]$. Because of the interest, we do not need to assume the net profit condition $\eta>0$.

Note that the process has deterministic paths between the claim times. Let $\left\{T_{k}\right\}$ denote the claim times. Then we have, for $t<T_{1}$,

$$
X_{t}^{B, m}=x+\int_{0}^{t} c\left(b_{s}\right)+m \int_{0}^{t} X_{s}^{B, m} \mathrm{~d} s,
$$

so that we can write

$$
X_{t}^{B, m}=\left(\int_{0}^{t} c\left(b_{s}\right) \mathrm{e}^{-m s} \mathrm{~d} s+x\right) \mathrm{e}^{m t} .
$$

Lemma 3.1. The function $V(x)$ is decreasing with $V(x)=0$ for $x \geq-c(0) m^{-1}$. It is Lipschitz continuous with $|V(x)-V(y)| \leq|x-y|$.

Proof. It is clear that $V(x)$ is decreasing. That $V(x)=0$ for $x \geq-c(0) m^{-1}$ follows as for the diffusion approximation.

Let $z>x$, and let $B=\left\{b_{t}\right\}$ be a reinsurance strategy for initial capital $z$ such that $V^{B}(z) \leq$ $V(z)+\varepsilon$. For initial capital $x$, choose the strategy $\tilde{B}$ (which is not optimal): inject the capital $z-x$ and then follow the strategy $B$. Thus,

$$
V(x)-V(z) \leq V^{\tilde{B}}(x)-V^{B}(z)+\varepsilon=z-x+\varepsilon .
$$

Because $\varepsilon$ is arbitrary we have $|V(x)-V(z)| \leq|x-z|$, which proves the Lipschitz continuity. As a consequence, $V(x)$ is absolutely continuous.

We conjecture that the value function $V(x)$ solves the HJB equation

$$
\inf _{b \in[0, \tilde{b}]} \lambda \int_{0}^{\infty} V(x-r(z, b)) \mathrm{d} G(z)+(c(b)+m x) V^{\prime}(x)-(\delta+\lambda) V(x)=0 .
$$

This is in fact the case. Let $b_{0}(x)$ be the argument for which the infimum is taken. The proof of the following result is similar to the proof of Theorem 3.2 of [4]. Details can be obtained on request from the authors. 
Theorem 3.1. The function $V(x)$ is differentiable from the right and from the left. Its derivatives solve (3.1). The strategy $B_{0}=\left\{b_{0}\left(X_{t}^{B_{0}, m}\right)\right\}$ is optimal, and $V(x)$ is continuously differentiable at all points $x$ where $c\left(b_{0}(x)\right)+m x \neq 0$. Moreover, if there exists a number $b$ such that $c(b) \geq \lambda \mathrm{E}[r(Z, b)]$ then any decreasing positive solution to (3.1) coincides with $V(x)$.

Remark 3.1. (Optimal strategy at $x=0$.) Suppose that the premium rate function $c(b)$ is calculated by the expected value principle:

$$
c(b)=\lambda(1+\theta) \mathrm{E}[r(Z, b)]-\lambda \mu(\theta-\eta) .
$$

Consider the initial capital $x=0$, and let $b_{0}$ be the root of the equation $c(b)=0$. Assume for the moment that a strategy $b$ with $c(b) \leq 0$ is optimal at $x=0$. Since the surplus never leaves the value 0 , we have

$$
V(0)=\mathrm{E}\left[\sum_{i=1}^{\infty} r\left(Z_{i}, b\right) \mathrm{e}^{-\delta T_{i}}\right]-\frac{c(b)}{\delta}=\frac{\lambda \mathrm{E}[r(Z, b)]-c(b)}{\delta} .
$$

From the HJB equation (3.1) we obtain, by rearranging the terms,

$$
V(0)=\frac{\lambda}{\delta}\left[\mathrm{E}[r(Z, b)]+\{(1+\theta) \mathrm{E}[r(Z, b)]-(\theta-\eta) \mu\} V^{\prime}(0)\right] .
$$

We conclude that $V^{\prime}(0)=-1$. This implies that the right-hand side of the above equation is decreasing in $b$; hence, $b=b_{0}$ would be optimal. In particular, $V(0)=\lambda \mathrm{E}\left[r\left(Z, b_{0}\right)\right] / \delta$. Let $\kappa, \varepsilon>0$ such that $\kappa>c(\tilde{b}) \varepsilon$. Consider the strategy $b_{t}=b_{0} \mathbf{1}_{\left\{t \geq T_{1} \wedge \varepsilon\right\}}$. This strategy has the value bounded by

$$
\begin{aligned}
& \mathrm{e}^{-(\lambda+\delta) \varepsilon}\left(\frac{\lambda}{\delta} \mathrm{E}\left[r\left(Z, b_{0}\right)\right]-\frac{\lambda}{\lambda+\delta} c(\tilde{b}) \varepsilon(1-G(\kappa))\right) \\
& +\int_{0}^{\varepsilon}\left(\mu+\frac{\lambda}{\delta} \mathrm{E}\left[r\left(Z, b_{0}\right)\right]-c(\tilde{b}) t(1-G(\kappa))\right) \lambda \mathrm{e}^{-(\lambda+\delta) t} \mathrm{~d} t .
\end{aligned}
$$

Taking the derivative with respect to $\varepsilon$ shows that the function is decreasing in $\varepsilon$, with a derivative bounded away from 0 . Thus, for small enough $\kappa$ and $\varepsilon$, the above strategy yields a smaller value than the strategy $b_{t}=b_{0}$. This shows that $b_{0}$ cannot be optimal.

Equation (3.1) at $x=0$ reads

$$
\inf _{b \in[0, \tilde{b}]} \lambda \mathrm{E}[r(Z, b)]\left[1+(1+\theta) V^{\prime}(0)\right]-\lambda \mu(\theta-\eta) V^{\prime}(0)-\delta V(0)=0 .
$$

We see that the minimum is taken either at $b=0$ or $b=\tilde{b}$. Because $b=0$ is not optimal, we conclude that $b=\tilde{b}$. In particular, we obtain $V^{\prime}(0)<-1 /(1+\theta)$ and $V^{\prime}(x)<-1 /(1+\theta)$ for $x \in[0, y)$ and some $y>0$. By the continuity of the left-hand side of (3.1), we conclude that $b^{*}(x)=\tilde{b}$ for small enough $x$. That is, no reinsurance is taken for capital close to 0 .

Remark 3.2. Consider the function

$$
g_{x}(b):=(c(b)+m x) V^{\prime}(x)-(\delta+\lambda) V(x)+\lambda \int_{0}^{\infty} V(x-r(z, b)) \mathrm{d} G(z) .
$$


Let $b_{1}, b_{2} \in[0, \tilde{b}]$ and $b_{1}>b_{2}$. Then from the Lipschitz continuity of $V(x)$ for every $x \in[0, \infty)$ we obtain

$$
\begin{aligned}
g_{x}\left(b_{1}\right)-g_{x}\left(b_{2}\right)= & \lambda \int_{0}^{\infty}\left(V\left(x-r\left(z, b_{1}\right)\right)-V\left(x-r\left(z, b_{2}\right)\right)\right) \mathrm{d} G(z) \\
& +\left(c\left(b_{1}\right)-c\left(b_{2}\right)\right) V^{\prime}(x) \\
= & \lambda \int_{0}^{\infty}\left(V\left(x-r\left(z, b_{1}\right)\right)-V\left(x-r\left(z, b_{2}\right)\right)\right. \\
& \left.+\left\{r\left(z, b_{1}\right)-r\left(z, b_{2}\right)\right\}(1+\theta) V^{\prime}(x)\right) \mathrm{d} G(z) \\
\leq & \lambda \int_{0}^{\infty}\left\{r\left(z, b_{1}\right)-r\left(z, b_{2}\right)\right\}\left[1+(1+\theta) V^{\prime}(x)\right] \mathrm{d} G(z) .
\end{aligned}
$$

The condition $V^{\prime}(x) \leq-1 /(1+\theta)$ implies that $g_{x}(b)$ is decreasing, so that the minimum is taken in $b=\tilde{b}$, which is then the optimal strategy if $V(x)$ is differentiable in $x$. On the other hand, if $b_{0}(x) \neq b<\tilde{b}$ is optimal for some $x \in[0, \infty)$ then it must hold that $V^{\prime}(x)>-1 /(1+\theta)$.

We did not establish that the value function is continuously differentiable, even though the authors believe that this is actually the case. We therefore now give a sufficient condition for continuous differentiability.

Lemma 3.2. If the value function $V(x)$ is convex then $V(x)$ is continuously differentiable.

Proof. Let $b_{0}(x)$ denote the root of the equation $c(b)+m x$ for $x \geq 0$, and define

$$
f(x):=\lambda \int_{0}^{\infty} V\left(x-r\left(b_{0}(x), z\right)\right) \mathrm{d} G(z)-(\delta+\lambda) V(x) .
$$

By (3.1), $f(x) \geq 0$. Let $V^{\prime}(x-)$ and $V^{\prime}(x+)$ denote the derivatives from the right and from the left, respectively. Assume now that there exists $\tilde{x} \in(0, \infty)$ with $V^{\prime}(\tilde{x}-)<V^{\prime}(\tilde{x}+)$. By Theorem 3.1, $f(\tilde{x})=0$. Since in 0 we only consider the derivative from the right, we have $\tilde{x}>0$. Without loss of generality, we can assume that $V(x)$ is continuously differentiable on $(0, \tilde{x})$, which means that $f(x)$ is continuously differentiable on $(0, \tilde{x})$. There exist sequences $\left(h_{n}\right)_{n \geq 0} \in(0, \tilde{x}), \lim _{n \rightarrow \infty} h_{n}=\tilde{x}$, with $f^{\prime}\left(h_{n}\right) \leq 0$ and $\left(x_{n}\right)_{n \geq 0} \in(\tilde{x}, \infty), \lim _{n \rightarrow \infty} x_{n}=\tilde{x}$, with $f^{\prime}\left(x_{n}\right) \geq 0$. Letting $n$ tend to $\infty$ we obtain

$$
\begin{aligned}
& \lambda \int_{0}^{\infty} V^{\prime}\left(\tilde{x}-r\left(b_{0}(x), z\right)\right) \mathrm{d} G(z)-(\delta+\lambda) V^{\prime}(\tilde{x}-) \leq 0, \\
& \lambda \int_{0}^{\infty} V^{\prime}\left(\tilde{x}-r\left(b_{0}(x), z\right)\right) \mathrm{d} G(z)-(\delta+\lambda) V^{\prime}(\tilde{x}+) \geq 0 .
\end{aligned}
$$

Thus, $V^{\prime}(\tilde{x}+) \leq V^{\prime}(\tilde{x}-)$, which is a contradiction.

In the examples below we consider the special case of proportional reinsurance.

\subsection{Examples}

Let us first note that in the case of proportional reinsurance we find, as in the case without an interest rate (see [4]), that the value function is convex, provided that $c(b)$ is concave. By Lemma 3.2 we can conclude that the value function is continuously differentiable. The problem in the numerical calculation of the value function is that we do not have the initial value $V(0)$. 
But since we know $V(x)=0$ for $x \geq \lambda \mu(\theta-\eta) m^{-1}$, the calculation of the initial value becomes less complicated than in the case without an interest rate. Solve (3.1) with the initial value $V_{0}$. Let us denote the corresponding solution by $f\left(x ; V_{0}\right)$. We then have to find $V_{0}$, such that $f\left(-m^{-1} c(0) ; V_{0}\right)=0$. If $V_{0}>V(0)$ then we would obtain $f\left(-m^{-1} c(0) ; V_{0}\right)>0$.

For a proof of the above statement, define

$$
g(x):=f\left(x ; V_{0}\right)-V(x) .
$$

If $V_{0}>V(0)$, we have $g(0)>0$. Let $b^{*}(x)$ denote the optimal strategy for $V(x)$, and let $b_{0}(x)$ be the root of the equation $c(b)+m x=0$. Replacing the optimal $b$ for $f(x)$ by $b^{*}(x)$ yields

$$
\left(c\left(b^{*}(x)\right)+m x\right) g^{\prime}(x)+\lambda \int_{0}^{\infty} g\left(x-b^{*}(x) z\right) \mathrm{d} G(z)-(\delta+\lambda) g(x) \geq 0 .
$$

Note that $g(x)=g(0)$ for $x \leq 0$. Because $g(0)>0$ and $b^{*}(0)=1$ (see Remark 3.1), it follows that $g^{\prime}(0)>0$. Let $\hat{x}=\inf \left\{x: g^{\prime}(x) \leq 0\right\}$. Because $g(x)$ is increasing, we conclude that $b^{*}(x)>b_{0}(x)$ on $[0, \hat{x})$. From (3.1) and Lemma 3.1, we conclude that $b^{*}(\hat{x})>b_{0}(\hat{x})$ also. Because $g(x)$ is increasing on $[0, \hat{x}]$, it follows from (3.2) that $\left(c\left(b^{*}(\hat{x})\right)+m \hat{x}\right) g^{\prime}(\hat{x})>0$, which is a contradiction. So the function $g(x)$ is strictly increasing on $\mathbb{R}_{+}$. Therefore, $f\left(x ; V_{0}\right)$ will ultimately be increasing.

Example 3.1. $(\operatorname{Exp}(1 / \mu)$ and Pareto-distributed claims.) For exponentially distributed claim sizes, we have to consider

$$
\inf _{b \in[0,1]} \frac{\lambda}{\mu} \int_{0}^{\infty} V(x-b z) \mathrm{e}^{-z / \mu} \mathrm{d} z+(\lambda \mu(b \theta-\eta)+m x) V^{\prime}(x)-(\delta+\lambda) V(x)=0 .
$$

The numerically calculated optimal strategy and value function are shown in Figure 3. The initial value is 1.37. For Pareto-distributed claims, the HJB equation becomes

$$
\inf _{b \in[0,1]} \lambda \int_{0}^{\infty} V(x-b z) \frac{2 \mu^{2}}{(\mu+z)^{3}} \mathrm{~d} z+(\lambda \mu(b \theta-\eta)+m x) V^{\prime}(x)-(\delta+\lambda) V(x)=0 .
$$

The numerically calculated optimal strategy and value function are given in Figure 4. The initial value is 1.91963 .
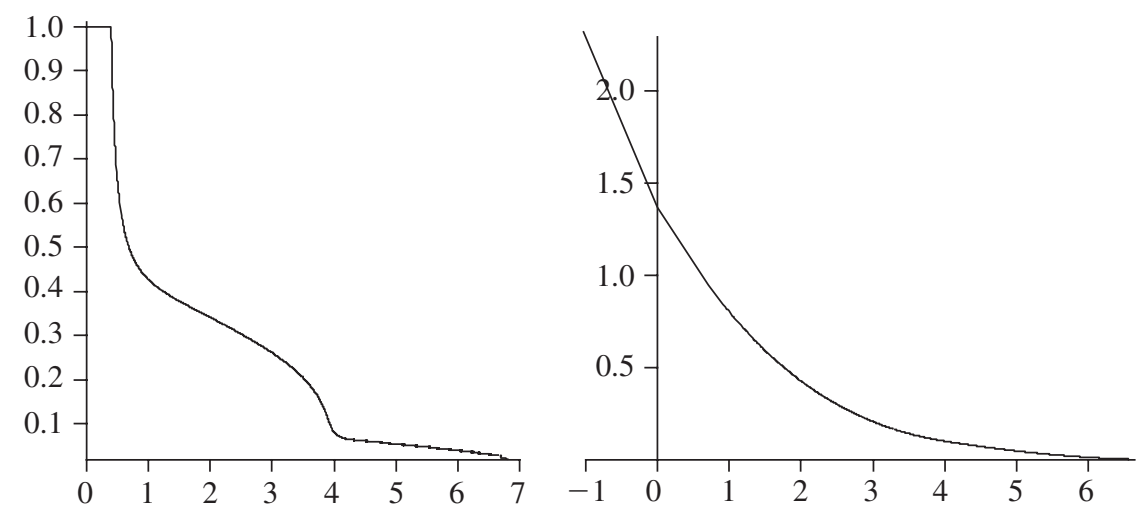

FIGURE 3: The optimal strategy (left) and the value function (right) for exponentially distributed claim sizes. 

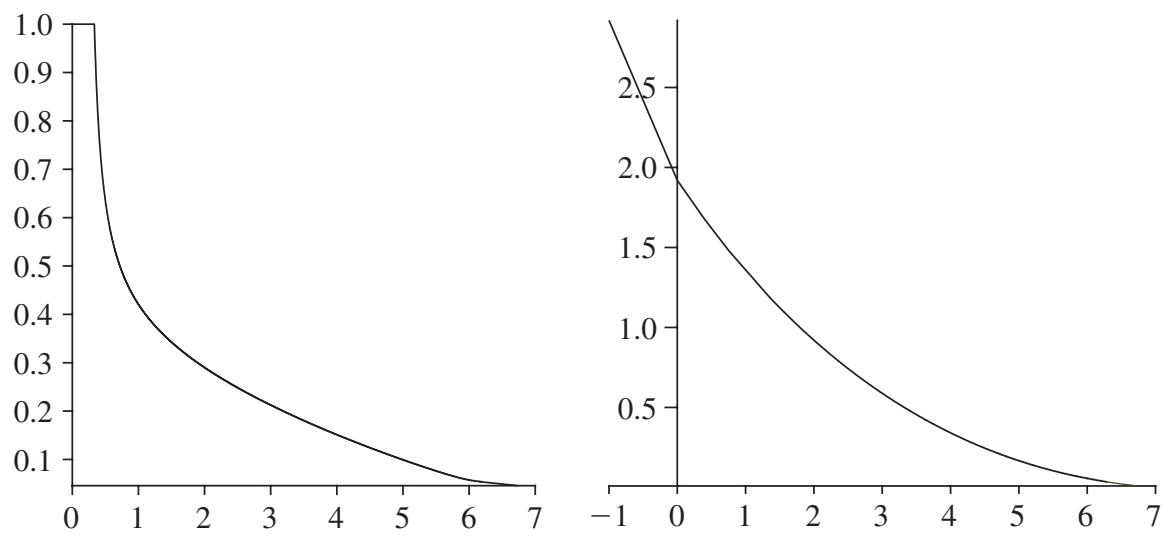

FIGURE 4: The optimal strategy (left) and the value function (right) for Pareto-distributed claim sizes.

Remark 3.3. For the special case $\delta=0$, we can show the existence of a strong (continuously differentiable) solution to the corresponding HJB equation

$$
\inf _{b \in[0, \tilde{b}]} \lambda \int_{0}^{\infty} V(x-r(z, b)) \mathrm{d} G(z)+(c(b)+m x) V^{\prime}(x)-\lambda V(x)=0
$$

via Banach's fixed point theorem. The starting point is to rewrite the HJB equation using Fubini's theorem, i.e.

$$
\lambda \int_{s(x, b)}^{\infty} r(z, b) \mathrm{d} G(z)-\lambda(1-G(s(x, b))) x=\lambda \int_{x}^{\infty}(1-G(s(y, b))) \mathrm{d} y,
$$

where $s(x, b)=\inf \{z: r(z, b)>x\}$. A detailed discussion of the topic for the classical risk model without the possibility to invest can be found in [4]. The proof techniques used there originate from [12, pp. 46-48]. We skip considering the $\delta=0$ case since the setup of the problem is similar to the setup described in [4]. We just remark that choosing $\delta=0$ facilitates the numerical calculation of the value function and of the optimal strategy considerably.

In the next example we give a numerical illustration.

Example 3.2. (Proportional reinsurance for $Z_{i} \sim \operatorname{Exp}(1 / \mu)$ and $Z_{i} \sim \operatorname{Pareto}(2, \mu)$.) We consider here only the proportional reinsurance, i.e. $r(z, b)=z b$.

All the considerations concerning the function $V^{1}(x)$ in the $\delta>0$ case also hold in the $\delta=0$ case. But here it is easier to consider the derivative $\left(V^{1}\right)^{\prime}$. For the exponentially distributed claim sizes, $Z_{i} \sim \operatorname{Exp}(1 / \mu)$, we have to solve the integro-differential equation

$$
-\lambda \int_{0}^{x}\left(V^{1}\right)^{\prime}(y) \mathrm{e}^{-(x-y) / \mu} \mathrm{d} y+c\left(V^{1}\right)^{\prime}(x)+\lambda \mu \mathrm{e}^{-x / \mu}=0 .
$$

This equation is easy to solve, and we obtain as the derivative

$$
\left(V^{1}\right)^{\prime}(x)=-\frac{\lambda \mu}{c}\left(1+\frac{m x}{c}\right)^{\lambda / m-1} \mathrm{e}^{-x / \mu}
$$

for $x \geq 0$. Choose $\mu=\lambda=1, m=0.03, \theta=0.5$, and $\eta=0.3$. We calculate the value function numerically. The value function and the optimal strategy for $Z_{i} \sim \operatorname{Exp}(1 / \mu)$ are given 

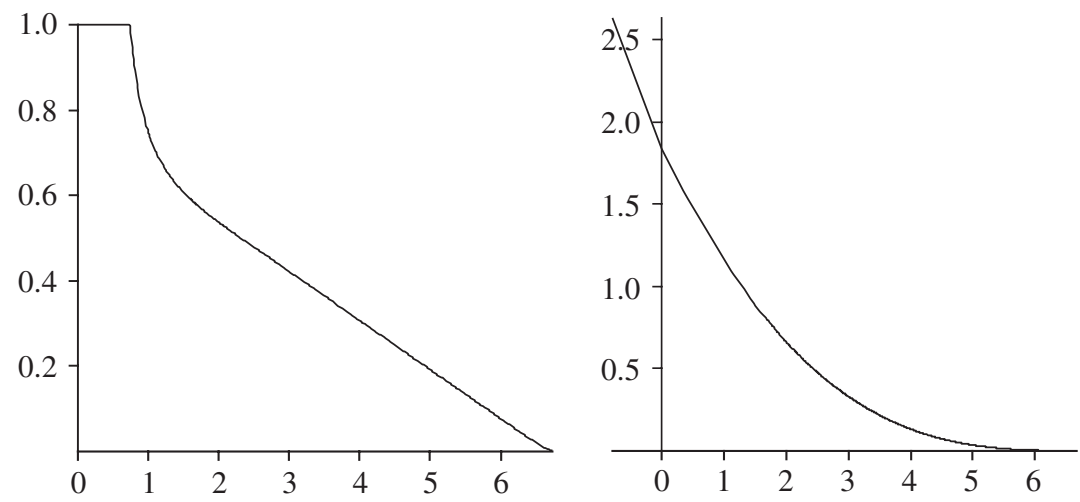

Figure 5: The optimal strategy (left) and the value function (right) for $Z_{i} \sim \operatorname{Exp}(1 / \mu)$.
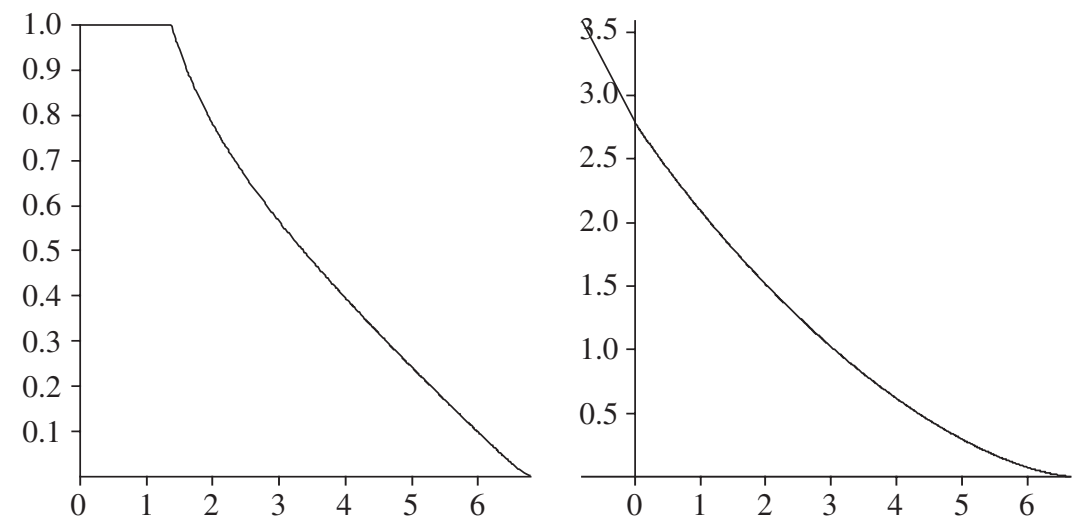

Figure 6: The optimal strategy (left) and the value function (right) for $Z_{i} \sim \operatorname{Pareto}(2, \mu)$.

in Figure 5. The value function and the optimal strategy for $Z_{i} \sim \operatorname{Pareto}(2, \mu)$ are shown in Figure 6.

\section{Acknowledgement}

The authors thank an anonymous referee for his/her helpful comments that improved the presentation of this paper.

\section{References}

[1] Asmussen, S. (2000). Ruin Probabilities. World Scientific, River Edge, NJ.

[2] Azcue, P. and Muler, N. (2005). Optimal reinsurance and dividend distribution policies in the CramérLundberg model. Math. Finance 15, 261-308.

[3] Eisenberg, J. And Schmidli, H. (2009). Optimal control of capital injections by reinsurance in a diffusion approximation. Blätter DGVFM 30,1-13.

[4] Eisenberg, J. AND SCHMidli, H. (2011). Minimising expected discounted capital injections by reinsurance in a classical risk model. To appear in Scand. Actuarial $J$.

[5] Grandell, J. (1991). Aspects of Risk Theory. Springer, New York.

[6] Hipp, C. AND Plum, M. (2000). Optimal investment for insurers. Insurance Math. Econom. 27, $215-228$.

[7] Hipp, C. AND Schmidli, H. (2004). Asymptotics of ruin probabilities for controlled risk processes in the small claims case. Scand. Actuarial J. 2004, 321-335. 
[8] Rogers, L. C. G. And Williams, D. (2000). Diffusions, Markov Processes, and Martingales, Vol. 2. Cambridge University Press.

[9] Rolski, T., Schmidli, H., Schmidt, V. And Teugels, J. (1999). Stochastic Processes for Insurance and Finance. John Wiley, Chichester.

[10] Schmidui, H. (2001). Optimal proportional reinsurance policies in a dynamic setting. Scand. Actuarial J. 2001, 55-68.

[11] Schmidli, H. (2002). On minimizing the ruin probability by investment and reinsurance. Ann. Appl. Prob. 12, 890-907.

[12] SchmiduI, H. (2008). Stochastic Control in Insurance. Springer, London.

[13] Schmidl, H. (2010). On the Gerber-Shiu function and change of measure. Insurance Math. Econom. 46, 3-11.

[14] Shreve, S. E., Lehoczky, J. P. AND Gaver, D. P. (1984). Optimal consumption for general diffusions with absorbing and reflecting barriers. SIAM J. Control Optimization 22, 55-75. 